\title{
CA \\ Social Characters: The Hierarchy of Gender in Contemporary English-Language Fiction
}

\author{
Eve Kraicer and Andrew Piper
}

01.30 .19

Peer-Reviewed By: Berenike Herrmann

Clusters: Gender

Article DOI: $10.22148 / 16.032$

Dataverse DOI: 10.7910/DVN/NFPJAQ

Journal ISSN: 2371-4549

Cite: Eve Kraicer and Andrew Piper, "Social Characters: The Hierarchy of Gender in Contemporary English-Language Fiction," Journal of Cultural Analytics. January 30, 2018.

Across a variety of cultural fields, researchers have identified a near ubiquitous underrepresentation and decentralization of women. This occurs both at the level of who is able to produce cultural works and who is depicted within them. Women are less likely to be directors of Hollywood films and also less likely to have starring roles. ${ }^{1}$ Women writers are less likely to be reviewed in major book review outlets as well as do the reviewing. ${ }^{2}$ Women are considerably less likely to

\footnotetext{
${ }^{1}$ Stacey L. Smith, Marc Choueiti, and Katherine Pieper, ”Inequality in 900 Popular Films, “; Geena Davis Institute on Gender and Media,"The Reel Truth: Women Aren't Seen or Heard: An Automated Analysis of Gender Representation in Popular Films" (2016). M. Erigha "Race, Gender, Hollywood: Representation in Cultural Production and Digital Media’s Potential for Change," Sociology Compass 9 (2015): 78-89; and Ellyn Rolleston Keith, "A Sentiment Analysis of Language \& Gender Using Word Embedding Models," CUNY Academic Works, (2017).

${ }^{2}$ For research on women and book-reviewing, see VIDA: Women in Literary Arts and CWILA: Canadian Women in the Literary Arts. The ratios in Canadian publishing/reviews are better than in the U.S. and UK (and approach parity), except in French-language reviews. For a discussion of thematic bias in book reviews even when women achieve parity, see Andrew Piper and Richard Jean
} 
be cited as sources within the news and be represented as authors in newspaper bylines. ${ }^{3}$ And women's lives are less likely to be written about in public forums like Wikipedia. ${ }^{4}$

Given the realities surrounding the underrepresentation of women within different contemporary cultural fields, we wanted to know more about women's place within contemporary fiction. Recent research by Ted Underwood, David Bamman and Sabrina Lee has found that while women writers were significantly underrepresented during much of the twentieth century, they have undergone a dramatic increase since the 1970s, achieving near parity by the turn of the twenty-first century. ${ }^{5}$ Rosie Cima has shown that similarly for best-selling fiction, women's share as authors has risen from a low of $14 \%$ in the mid-1970s to near-parity today. ${ }^{6}$ Despite continued marginalization in book reviews, then, it appears that women writers, especially of fiction, have defied larger cultural trends.

And yet as our research will endeavor to show, when we look at the representation of women within fiction-that is when we look at how women are characterized-we find familiar patterns of marginalization. Analyzing a set of 26,450 characters from 1,333 novels published between 2001 and 2015 from seven different genres (prizewinners, bestsellers, books reviewed in the New York Times, mystery, science fiction, romance, and young adult), we examine the social positions of women as fictional characters, ranging from their overall visibility or presence in novels to different types of social connectivity. In doing so, we find four patterns that merit further discussion:

So, "Women Write About Family, Men Write About War," The New Republic April 8, 2016. For a comprehensive, computationally driven study on author gender and reception in the Dutch context, see Corina Koolen, Reading beyond the Female: The relationship between perception of author gender and literary quality (Amsterdam: ILLC Disertation Series, 2018).

${ }^{3}$ Women's Media Center, "The Status of Women in the U.S. Media,“; Eran Shor, Arnout van de Rijt, Alex Miltsov, Vivek Kulkarni, and Steven Skiena,"A Paper ceiling: Explaining the persistent underrepresentation of women in printed news," American Sociological Review 80, no. 5 (2015): 960984. For bias in the language of journalism, see Liye Fu, Cristian Danescu-Niculescu-Mizil, Lillian Lee, "Tie-breaker: Using language models to quantify gender bias in sports journalism," from the proceedings of the 2016 IJCAI workshop on NLP meets Journalism.

${ }^{4}$ Claudia Wagner, David Garcia, Mohsen Jadidi, Markus Strohmaier, "It's a Man's Wikipedia? Asssessing Gender Inequality in an Online Enyclopedia," The International AAAI Conference on Web and Social Media (ICWSM2015). It is important to point out that the authors find that based on external registers of "notable people" women are not less likely to be written about. However, the overall prevalence of women's biographies is considerably below men's on Wikipedia. Additionally, the authors find significant lexical differences between the two groups.

${ }^{5}$ Ted Underwood, David Bamman, and Sabrina Lee, "The Transformation of Gender in EnglishLanguage Fiction," Cultural AnalyticsFebruary 12, 2018. DOI: 10.7910/DVN/TEGMGI

${ }^{6}$ See Rosie Cima "Bias, She Wrote," The Pudding (2017). 
First, as with other cultural fields, we find that our corpus favours men over women, with a roughly 62:38 ratio of men to women for all major characters. This level approaches the 2:1 ratio that we often see in other domains (which we might call the "golden mean of patriarchy"). The exception to this rule, as we will see, are protagonists. When looking only at this most prominent character, we find a 50:50 ratio of men and women characters. This suggests that while efforts have been made to address gender bias for leading characters of novels, the skew towards men still occurs among the rest of the characters. Below the surface of protagonism, the systemic biases of gender norms reappear.

Second, we find that along with this prevalence of men in fiction, contemporary novels favour heteronormative pairings - interactions between men and women - over pairings between characters of the same gender, a fact that has not previously been explored in other domains. While the term heteronormativity is usually reserved for sexual pairings-the assumption that heterosexuality is the default form of human sexuality to the exclusion of all other forms-we use it hear to indicate the extent to which mixed-gender pairs, whether sexual or platonic, significantly dominate the contemporary novel's social structure.

Third, we find that the prevalence of gender bias and heteronormativity occur in highly standardized ways across genres. The hierarchy of character gender and the standard of heteronormative interactions appear to transcend very different kinds of writing and readerships, pointing to something more systemic at work in these narrative structures.

Finally, our research indicates that although books written by women temper the strength of these biases, women authors nonetheless maintain a tendency towards featuring and populating their narratives with more characters that are men, and are even more likely to create heteronormative social networks.

We feel this research is important because it allows us to understand the largescale imagination of gender norms in fiction in addition to the actual roles of who gets published or who gets reviewed. Taking the Geena Davis Institute on Gender and Media's credo as our guiding motto, "If she can see it, she can be it," we subscribe to the belief that fictional representations have an important social function in positing social possibilities. ${ }^{7}$ New tools like David Bamman's BookNLP, which is our primary resource for studying character here, provide researchers

\footnotetext{
${ }^{7}$ An interesting line of empirical work could investigate the association between fictional and realworld representations in various cultural domains. For a recent example, see Beate Volker and Roel Smeets, "Mirrors or alternative worlds? Networks of characters in contemporary Dutch literature compared to actual networks." Long paper at International Sunbelt Social Network Conference 2018 (International Network for Social Network Analysis), 30-6-2018.
} 
with the ability to study the process of characterization at large-scale. ${ }^{8}$ As Underwood, Bamman, and Lee have shown, the semantic distinctions between genders within novels appear to be declining over time, suggesting that gender norms are becoming more porous in relation to character attributes and behavior. ${ }^{9}$ And yet when we focus on the prominence and connectivity of characters, that is, when we look at characters within their social context within novels, we see how such semantic dedifferentiation is offset by the persistence of hierarchical gender norms.

In what follows, we begin with a discussion of the data we used for our study, the steps we took to prepare it for analysis, and an analysis of the error associated with the gender predictions used throughout. As we will emphasize, it is important to note that all of our findings are based on estimates of predicted gender, not actual gender assignments (we will return to the limitations of working with binary assignments at the close of the essay). We then describe two primary ways of studying what we are calling the social context of characters. The first section investigates "visibility" by measuring the prevalence of women-identified characters, ranked by the rate at which they are mentioned, and how those values interact with genre and author gender. In the second section, we explore "connectivity" by measuring gender configurations either in pairs or trios, where we define a connection as a co-occurrence between characters at the sentence level. We use the social network measure of assortativity to assess heteronormativity within novels, i.e. the likelihood of characters being paired with the opposite gender, and then conclude by testing the association of gender with social balance theory with respect to triangular character relationships. We close with a discussion of the theoretical limitations of our data and methods, as well as what we see as the potential implications of our findings.

\section{Data Set}

For this study, we use a collection of 1,333 contemporary novels published between 2000 and 2015 that are drawn from seven different genres, where genre is defined as both a thematic category (young-adult, mystery, science fiction, romance) and a category of social distinction (bestsellers, prizewinners, and novels

\footnotetext{
${ }^{8}$ See David Bamman, Ted Underwood and Noah Smith, "A Bayesian Mixed Effects Model of Literary Character," In Proceedings of the 52nd Annual Meeting of the Association for Computational Linguistics (2014): 370-379.

${ }^{9}$ Underwood, Bamman, and Lee, “The Transformation of Gender in English-Language Fiction.”
} 
reviewed in the New York Times). ${ }^{10}$ To collect titles for our thematic categories, we used Amazon.ca with respect to each of the corresponding genre tags on Amazon. To select titles, we sorted by popularity within a genre tag and then selected the highest ranked novels. For YA, we combined our Amazon-selected books with Readers' Choice Awards from Goodreads.com. To collect titles for our social distinction categories, we used three methods: for bestsellers, we collected the top 200 titles that had appeared for the most number of weeks on the New York Times bestsellers list since 2000 in descending order. For prizewinners, we collected all shortlisted and winning titles from five major literary awards in the US, UK, and Canada (the Giller Prize, Governor General's Award, Pen/Faulkner, National Book Award, and Man Booker Award). Finally, for NYT Reviewed, we collected novels reviewed by the New York Times during our time frame. Table 1 shows the breakdown of our texts by genre and author gender. ${ }^{11}$

\begin{tabular}{lllll}
\hline Genre & Code & Novels & \# Authors & \% Women Authors \\
\hline Science Fiction & SF & 192 & 155 & 31.8 \\
Prizewinners & PW & 208 & 188 & 41.4 \\
Bestsellers & BS & 195 & 96 & 41.5 \\
NYT Reviewed & NYT & 180 & 179 & 48.9 \\
Mystery & MY & 188 & 140 & 51.6 \\
Young Adult & YA & 174 & 144 & 84.5 \\
Romance & ROM & 196 & 172 & 98.5 \\
\hline
\end{tabular}

Table 1. Summary of our data, with the number of works per genre, number of authors. Genres are ranked by percent of women authors per genre.

While this collection should in no way be taken as a definitive representation of contemporary publishing as a whole, our aim has been to capture different types of well-selling writing with respect to narrative theme and audience-type that have passed through professional publishing filters. It gives us a perspective on how different genres and books geared to different readerships that have sold well behave with respect to gender over the past decade and a half. At the same time, our data is limited to a representation of professional publishing and thus cannot address the growing world of self-publishing and online user-generated fiction. Given the increasing popularity and substantial growth in these areas, future research should be devoted to better understanding their differences with published fiction. ${ }^{12}$ In order to account for the overall heterogeneity of our corpus, i.e. to test whether there are significant internal differences between the dif-

\footnotetext{
${ }^{10}$ A similar version of this data set was used in Andrew Piper and Eva Portelance, "How Cultural Capital Works: Prizewinning Novels, Bestsellers, and the Time of Reading," Post45, May 10, 2016.

${ }^{11}$ Five authors were not able to be identified by gender in our analysis.

${ }^{12}$ For an initial study, see Smitha Milli and David Bamman, "Beyond Canonical Texts: A Computational Analysis of Fanfiction," in Proceedings of the 2016 Conference on Empirical Methods in Natural Language Processing, Austin, Texas, November 1-5, 2016, 2048-2053.
} 
ferent genre categories, we use two tactics: first, we use the method of bootstrap sampling throughout to estimate our various measures, and second, we also report the effects that genre and author gender have on our findings to account for any internal differences that may exist within our sample. It should be noted that for all measurements related to author gender, we remove Romance because it is so heavily skewed towards a single gender.

\section{Data Preparation}

To prepare our data for analysis, we apply David Bamman's BookNLP to all of our texts in order to perform the following tasks: a) named entity recognition to identify persons within the text; b) map entities and their aliases (nicknames as well as pronouns) to a single character ID (anaphora resolution); c) mark character occurrences by sentence boundaries; and d) assign a predicted gender to each character. ${ }^{13}$ This allows us to extract a list of predicted characters ranked by their number of mentions within every novel and a predicted gender, which can include one of three possible options (M, F, and ? for unknown). ${ }^{14}$ The outputs of these features are referred to in our accompanying data as our "node lists" and serve as the basis of our analysis in the visibility section.

Second, from this data we generate tables of character co-occurrence, where every instance of two characters co-occurring within the boundaries of a sentence are registered as an undirected pair. Additionally, we use the Bing Sentiment dictionary to record a sentiment score for every sentence in which characters cooccur. ${ }^{15}$ These tables are referred to as our "edge lists" in the accompanying data and serve as the basis of our analysis in the "connectivity" section.

Because we are using predictions of character gender, we take two steps to ad-

\footnotetext{
${ }^{13}$ See David Bamman, Ted Underwood and Noah Smith, "A Bayesian Mixed Effects Model of Literary Character." Proceedings of the 52nd Annual Meeting of the Association for Computational Linguistics (2014): 370-379.

${ }^{14}$ BookNLP outputs a list of characters based on the occurrences of their proper names. We calculate by contrast the occurrence of all resolved instances of each character's mention, which includes pronominal references as well. For gender prediction, BookNLP assigns a gender label through a two step process: first it measures the ratio of masculine and feminine pronouns associated with a character and assigns a label based on a majority of the pronouns. Where no pronouns have been resolved to a particular character, it uses a dictionary of proper names to assign a gender label. Finally, where neither approach yields a label, it provides a label of "?" (unknown).

${ }^{15}$ Bing Liu, "Sentiment Analysis and Subjectivity," Handbook of natural language processing 2 (2010): 627-666.
} 
dress potential error within our analysis. First, for all but one of our measures we focus only on the top twenty characters for a given novel. We know for example that character prominence declines non-linearly as a function of rank (Figure 1). By the twentieth character we are encountering characters that average about 42 mentions total throughout a novel. Second, as we can see in Figure 2, the percentage of non-assigned genders rises considerably as we descend in character rank. Within the top twenty characters, only $11.5 \%$ of characters (or just slightly more than 2 per novel) are not labeled. Within the top ten it drops to $5.9 \%$. Given the high degree of non-assignment as we move out from the main list of characters, any conclusions about the effect of gender across all characters needs to be handled with a great deal of caution.

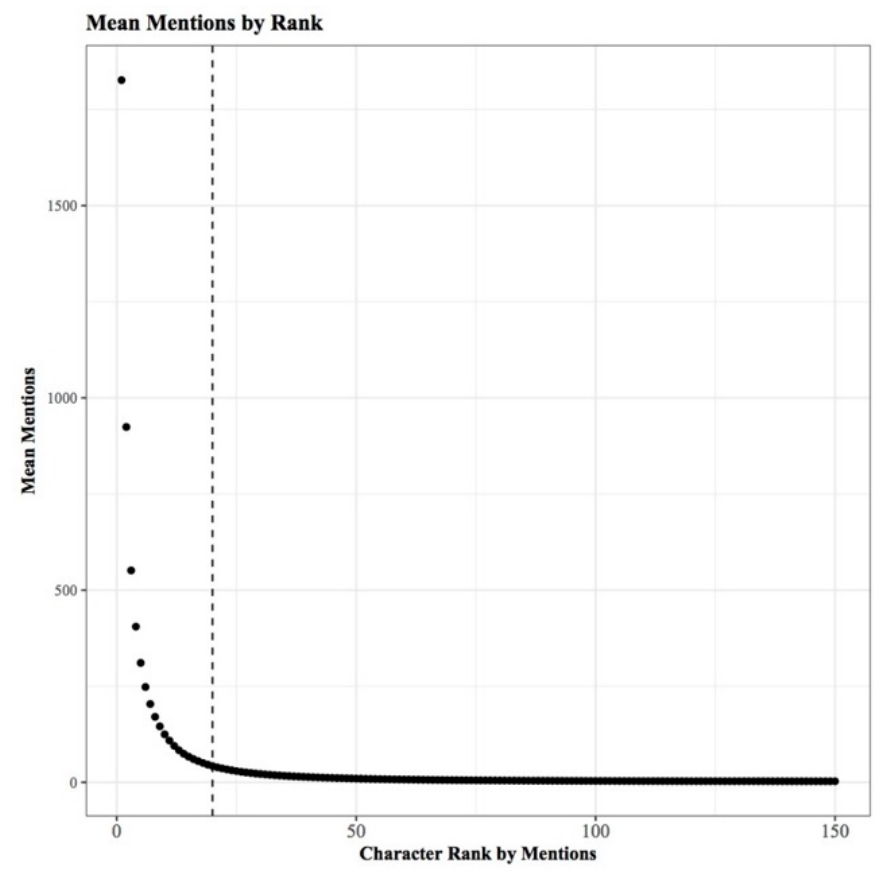

Figure 1. Number of character mentions by rank. Dotted line represents the twentieth ranked character. 


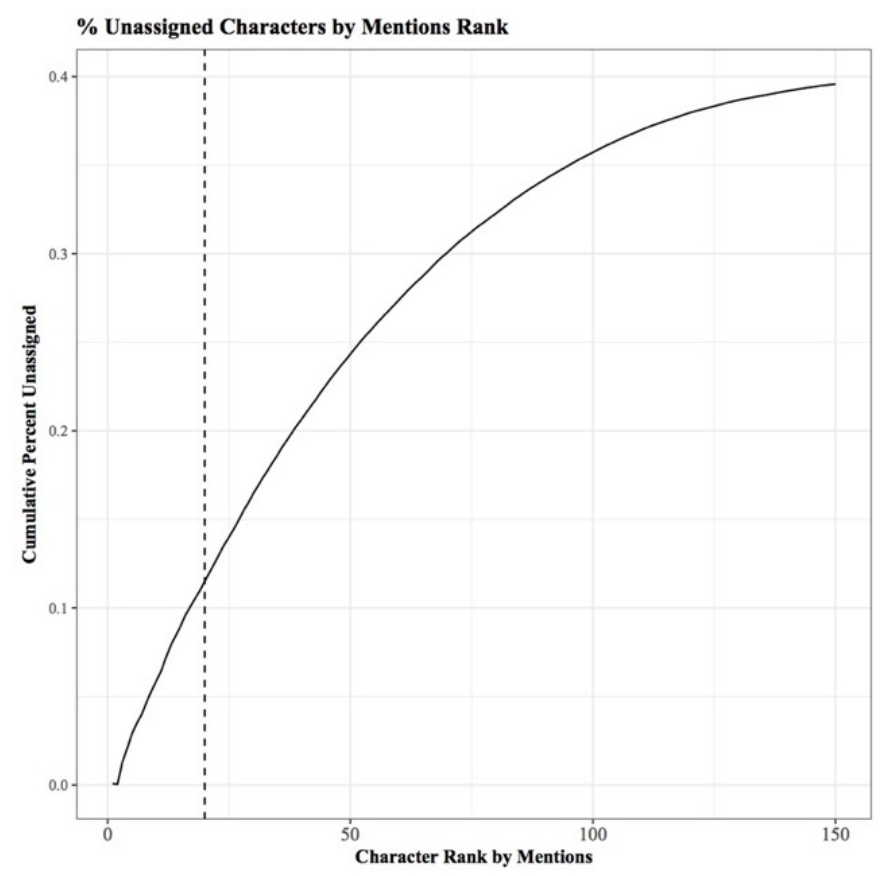

Figure 2. Percent of unassigned gender by character rank. Dotted line equals the twentieth ranked character.

Even within the top-twenty characters in a book, however, some gender assignments are inaccurate. To validate this, we manually verified Book NLP's predicted genders on two separate samples of two-hundred characters each: the first was only drawn from the top two characters from every book, where there were no non-assigned genders, while the second was drawn from the top twenty characters, which contained a proportion of non-assigned genders. ${ }^{16}$ We then calculated the sensitivity (or true positive rate) and specificity (the true negative rate) for each of our validation samples. Sensitivity captures the proportion of positives that are correctly labeled-in this case how many actual women are identified as women. Specificity captures the proportion of negatives correctly labeled as such-in this case how many men are labeled as not a woman. We found a sensitivity of $83.75 \% / 78.57 \%$ for women in the top two / twenty characters respectively and a rate of $96 \% / 96.9 \%$ for men, suggesting that women characters

\footnotetext{
${ }^{16}$ Each sample consisted of two hundred characters, where one-hundred were drawn randomly from the corpus and one hundred were drawn equally from our seven genres. All validation tables are included in the supplementary data.
} 
are potentially being undercounted in our data.

In order to account for this error, we modify our estimates of gender throughout using Rogan and Gladen's approach to estimating the true prevalence of a phenomenon given known errors in its detection. ${ }^{17}$ This approach adjusts the actual prevalence in a given sample according to the sample size and amount of error in the specificity and sensitivity of the predictions and is often used in epidemiology to estimate the true prevalence of a disease given known screening errors. For example, we don't know how many people have a disease, but after screening a sample of the population we find $\mathrm{X} \%$ of positive cases, but we know there is a certain degree of error associated with the screening. Rogan and Gladen's method adjusts the estimated "prevalence" of the disease using these error estimates. In our case the observed number of women-identified characters in our corpus was 8,362 or $31.6 \%$ (referred to as the "actual prevalence"). Using Rogan and Gladen's adjustment, we then estimate the true prevalence to be 10,003 or $37.8 \% .{ }^{18}$ As a further step, we use bootstrap sampling to estimate the overall variance of this estimated true prevalence of women characters. For every measure, then, we calculate the bootstrapped mean and standard deviation to assess how much within-sample variance there is. The net effect of both of these tactics is to elevate our estimates of the presence of women in novels from the raw counts produced by our BookNLP data.

\section{Visibility}

How visible are women in novels? There are different ways in which one might try to answer this question. We conceptualize the visibility of women in novels as a problem of hierarchical ranking-who gets mentioned more in a novel and how is the gender of characters distributed in terms of the frequency of mentions? Rather than look at overall ratios of gendered pronouns for example, we are interested in the ranked ordering of men and women as entities within a novel's fictional universe. To measure this, we rank characters by the number of mentions a character receives over the course of a novel as detected by BookNLP

\footnotetext{
${ }^{17}$ Walter J. Rogan and Beth Gladen, "Estimating Prevalence From The Results Of A Screening Test," American Journal Of Epidemiology107:1 (1978): 71-76, Doi.Org/10.1093/Oxfordjournals.Aje.A112510. We implement this using the epi.R package in R.

${ }^{18}$ These are values based only on the top twenty characters for each novel. See below for further description of this subset.
} 
(including proper names, aliases, and pronouns associated with characters) and then assign that character a gender based on BookNLP's prediction. We then calculate social visibility as the ratio of genders across four related sets: all characters; the top twenty most important characters; the novel's main character; and finally the top-pair of characters. In addition to comparing these distributions across genres, we also examine the extent to which women authors influence the visibility of women characters in novels. These measures are designed to mirror previous research on women's underrepresentation in other social and cultural spaces.

\section{Top 20 and All}

We estimate the prevalence of women characters in the top twenty characters of novels to be $37.8 \%$ for all genres (+/- $0.3 \%)$. This estimate is based on using 10,000 bootstrapped samples from an overall sample of 26,540 characters whose gender prediction has been adjusted using Roden and Gladen's method described above. Table 2 breaks down this overall average by genre. A chi-square test of independence on the estimated counts by genre suggests that the level of women characters is not significantly dependent on different genres and readerships $\left(\mathrm{X}^{2}(6\right.$, $\mathrm{N}=26,450)=7.6652, \mathrm{p}=0.2637$ ). However, the level of women characters is strongly associated with the gender of the author $\left(\mathrm{X}^{2}(1, \mathrm{~N}=22,602)=43.587, \mathrm{p}=\right.$ 4.1e-11), with books by women having $18 \%$ more women characters than books by men. With the exception of the top character (which we will discuss next), however, despite using more women characters, women authors never do so to the point where women characters assume a majority position at any other rank except the first (Fig. 3 and 4).

\begin{tabular}{ll}
\hline Genre & \% Women Top-Twenty Characters \\
\hline BS & 36.1 \\
PW & 36.45 \\
NYT & 36.9 \\
MY & 36.93 \\
SF & 37.7 \\
ROM & 38.2 \\
YA & 40.1 \\
\hline
\end{tabular}

Table 2. Estimated percentage of women in the top twenty characters by genre. 


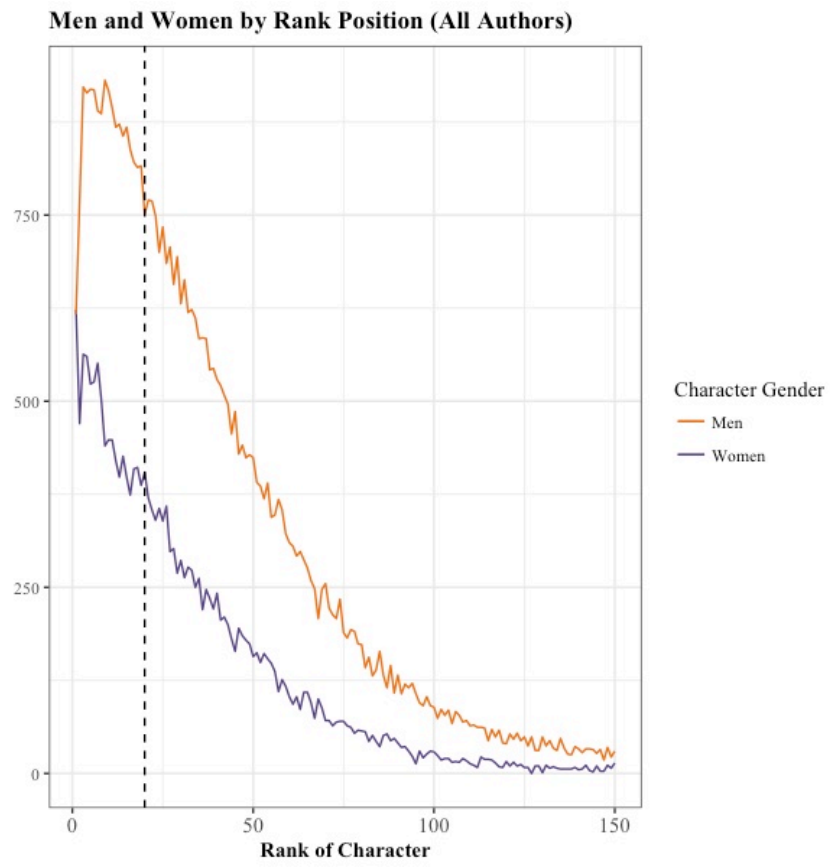

Figure 3. Count by character rank for all authors. 


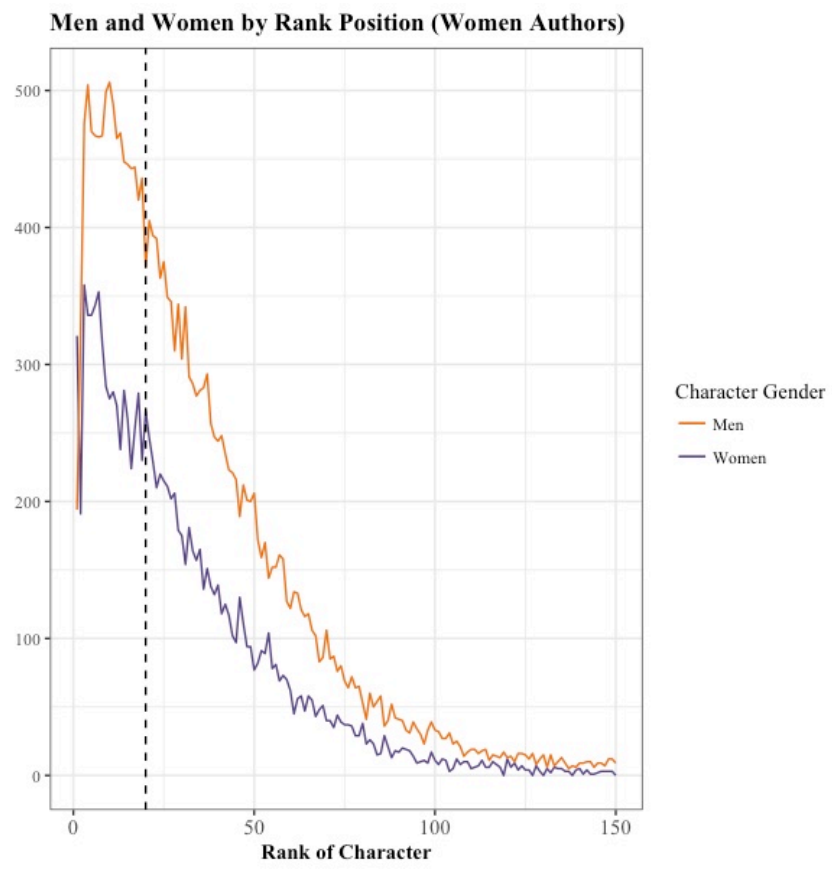

Figure 4. Count by character rank for women authors.

\section{Main Character}

While women are significantly less well-represented in our corpus regardless of the author's gender, this finding does not hold true for the main character of novels. The analysis of main character gender is made somewhat trickier, however, because of the fact that BookNLP is not currently able to map a book's narrator onto the appropriate character in the case of first person novels, or more technically speaking, novels with "character-bound" or "homodiegetic" narrators (as opposed to "external" or "heterodiegetic" narrators). In order to divide our corpus by point of view and predict the gender of homodiegetic narrators, we undertake the following steps. First, we develop a classifier that predicts whether a book has a homo- or heterodiegetic narrator (first or third person). ${ }^{19}$ Our method cur-

\footnotetext{
${ }^{19}$ To do so, we remove dialogue from novels and use two variables: pronoun counts and the bigram 'I said'. Because the label of homodiegetic results in IS? a separate method for identifying the main
} 
rently achieves an accuracy of $93.2 \%$, based on a training sample of 200 novels. Doing so we find that 664 novels in our corpus are homodiegetic and 669 are heterodiegetic. Second, we then developed a method that matches the character of a homodiegetic novel with the novel's narrator using the BookNLP data. ${ }^{20}$ This step yields a sensitivity of 0.75 and a specificity of 1.0 based on a test sample of 100 novels. We then build this error into our estimation of the true prevalence of women protagonists in homodiegetic works once again using Rogan and Gladen's method. Through this process, protagonist genders were identified in 1,239 works from our corpus. The remaining cases ( 94 works) were removed due to an unknown gender in the protagonist position or corruption of outputs during data processing of the homodiegetic works.

Based on this method, we find that the estimated prevalence of women main characters across our entire sample is $50.2 \%$, with a slightly higher amount in homodiegetic novels (54.6\%) and a slightly lower amount in heterodiegetic novels $(45.6 \%)$. When we subset the data by genre (Table 3 ), despite the relatively high degree of variation a chi-square test still indicates that protagonist gender is not significantly associated with genre $\left(X^{2}(6, N=1239)=9.9579, p=0.1264\right)$. Author gender, however, has a significant association with the distribution of main characters. In books written by women, $62.3 \%$ of books centralize a woman as the main character, while only $34.2 \%$ of books written by men do. In other words, women are 1.83 times more likely to write a woman main character than men authors $\left(\mathrm{X}^{2}(1, \mathrm{~N}=1048)=28.69, \mathrm{p}=8.496 \mathrm{e}-08\right)$.

\begin{tabular}{ll}
\hline Genre & \% Women \\
\hline PW & 43.3 \\
SF & 45 \\
NYT & 45.1 \\
MY & 47.3 \\
BS & 47.9 \\
YA & 61.4 \\
ROM & 63.1 \\
\hline
\end{tabular}

Table 3. Estimated gender of main characters by genre.

character of a novel we err? on the side of caution: we set the threshold for predicting a homodiegetic novel to a probability of 0.6 and above. With this threshold, we achieve a precision of .95 and a recall of .89 .

${ }^{20}$ Here we condition on characters who have the highest chance of appearing in a NULL dependency relationship. What is this? Please specify. It is based on the intuition that protagonists who are narrators are special types of characters who are more often the object of other people's thoughts, comments or actions and who less often engage as proper names in agential behavior (but rather are referred to by first person pronouns). 


\section{Top Pairs}

While protagonists are the most visible of all characters, and thus matter to a novel's plot, the top pair of characters is also an important analytical frame. The top pair represents a novel's primary relationship, and can encode important information about the novel's dramatic structure. While we will further explore this notion of pairing (and conflict) in the next section, here we want to better understand the gendered nature of leading relationships in novels. First, we find that the most common pairing of top characters is mixed gender (Table 4), accounting for $51 \%$ of all pairs. Second, if we look at same gendered pairs, we see that women-only pairs comprise just $18.6 \%$ of all top pairs in our data, while for men-only it is $30.4 \%$. When the protagonist is a man, in other words, readers are more likely to see a second leading man than a second leading woman when the protagonist is a woman. Over half of all top pairs that have a protagonist that is a man also have a man in the second position (61.2\%), while well under half of all top pairs with a woman as protagonist have a second woman character (37.2\%).

The pattern between men and women as same gender top pairs, as with main character, is strongly consistent across genre $\left(\mathrm{X}^{2}(6,1239)=4.2024, \mathrm{p}=\right.$ 0.6493). As with the main character, this too is dependent on author gender: given a same gendered pair, authors that are men are 1.6 times more likely to write two leading men $(\mathrm{p}=0.001034)$, while women authors are 2.1 times more likely than men authors to write leading women pair $(\mathrm{p}=8.342 \mathrm{e}-6)$. This means that although both groups write more of their own gender pairs, the extent to which the authors demonstrate this skew is unequal. Put another way, books by women have top pairs of men much more often ( $21 \%$ of all books by women), than books by men have top pairs of women (13.3\% of all books by men).

\begin{tabular}{lll}
\hline Genre & \% Same Gender Top Pairs & MM / WW Ratio \\
\hline BS & 49.9 & 1.2 \\
YA & 45.4 & 1.3 \\
MY & 50.7 & 1.5 \\
PW & 51.1 & 1.7 \\
ROM & 43.6 & 1.8 \\
SF & 51 & 1.9 \\
NYT & 51.6 & 2.1 \\
\hline
\end{tabular}

Table 4. Estimated percentage of same-gender top pairs in novels as well as the ratio of men-only to women-only top-pairs by genre. 


\section{Connectivity}

While the previous measures indicate the underrepresentation of women in fiction as individual entities, we are also interested in the ways gender influences interactions between characters. While there has been research on real-world social networks as they relate to gender, very little work has been done on the place of gender within fictional social networks. ${ }^{21}$ We explore the effect of gender on social networks in the novel using three measures. First, we analyze the structure and variation of gender assortativity in our corpus. Are men and women more likely to interact with characters of the same or different gender? Second, we use sentiment scores to look at "antagonism," asking how different gender pairings influence positive and negative language surrounding the co-occurrence of characters. Finally, we also use sentiment scores to analyze the social "balance" in gender configurations of social triangle. As with measures of visibility, we investigate how these trends are constructed across both genre and author gender.

For our purposes, we define an "interaction" as the co-occurrence of two characters within the boundary of a sentence. ${ }^{22}$ A "relationship" is defined as a connection between any two characters regardless of the number of interactions. A "weighted relationship" defines a connection where the number of interactions is used to account for the intensity or strength of that relationship in the novel. Thus, characters who co-occur often will have a stronger weighted relationship than characters who co-occur less frequently.

\section{Gender Assortativity}

Assortativity examines the extent to which connections in a social network are being made between nodes of similar quality or value. In our case, we explore the likelihood that nodes of the same gender are connected. The assortativity coefficient can be thought of as similar to a correlation coefficient comparing the

\footnotetext{
${ }^{21}$ For work in this direction, see Walt Hickey, "The Dollar-And-Cents Case Against Hollywood's Exclusion of Women," FixeThirtyEight (2016); Scott Selisker, “The Bechdel test and the Social Form of Character Networks," New Literary History 46, no. 2 (2015): 5050-523; and Volker, Beate \& Roel Smeets, "Mirrors or alternative worlds? Networks of characters in contemporary Dutch literature compared to actual networks," Long paper at International Sunbelt Social Network Conference 2018 (International Network for Social Network Analysis), 30-6-2018.

${ }^{22}$ For recent work that uses sentence co-occurrence of characters to classify novels using social network measures, see Mariona Coll Ardanuy and Caroline Sporleder. "Clustering of Novels Represented as Social Networks," LiLT (Linguistic Issues in Language Technology) 12 (2015).
} 
dependence of two variables, similarly falling between -1 and 1 . A positive value indicates that a greater number of connections are between characters of the same predicted gender, while a negative number indicates a greater degree of mixedgender relationships or interactions. We test assortativity by both relationships and interactions, meaning we look at the overall number of relationships between characters as well as the number of interactions that comprise those relationships (i.e. weighted relationships). Because we can only compute assortativity with positive labels, we remove all characters without an assigned gender from this section. As we can see in Fig. 5 and Fig. 6, across all genres assortativity tends to be negative for character relationships and interactions, and becomes more strongly negative when we look at more important characters (the top ten versus the top twenty versus all). ${ }^{23}$

Two things are notable here. First, while the majority of novels favor mixedgender pairs, there are very strong outliers that indicate more same-gender pairings (which appears to be strongest for example in the prizewinners category). Of the six prizewinning novels that exhibit very high assortativity, four have more man-man relationships than woman-woman relationships. Second, some of the effect of disassortativity we are seeing (the overall negative score) is due to the imbalanced gender distribution of characters in novels. When there are fewer women than men in novels, it is more likely that women will be paired with men because there are fewer women with whom they can engage. Assortativity is in part dependent on the balance of the classes.

Nevertheless, we can also test the extent to which these pairings exceed what we might see by chance. In other words, despite the overall bias towards more men in novels is the same-gender pairing in novels just a random effect of how many men and women there are in novels or is it something we might indicate as more "intentional" on the part of authors (even if those intentions are not fully conscious to authors)? In order to test this, we conduct a permutation test on our data to estimate the likelihood of finding this degree of disassortativity given the same ratio of men and women characters and the same number of interactions. To do so, we randomly shuffle the gender assignments for characters in novels and recalculate the average assortativity score for all novels 1,000 times for both relationships and interactions.

Doing so, we find that the average disassortativity for interactions is significantly greater than expected-not only does it exceed $95 \%$ of all permutations, it is

\footnotetext{
${ }^{23}$ We adjust each novel's distribution of character genders based on Roden and Gladen's method here as well. To do so, we randomly reassign the appropriate number of gender labels based on the estimated true prevalence adjustment and then calculate the assortativity. We do this 1,000 times for every novel and use the average assortativity for every novel as our estimated values.
} 
greater than even the lowest random permutation. However, this is not the case for relationships, where we see no significant deviation between the observed degree of disassortativity and a random distribution. In other words, at the level of plotting relationships (who is connected to whom), we see nothing of significance, whereas when we look at the level of interactions, of how often those characters are connected, we see a very strong effect. When we randomly shuffle the gender assignments of our characters, then, we never find the degree of mixed pairing at the sentence level that we find in our contemporary novels, even when we keep the overall gender ratios constant, suggesting that there is a deliberate orientation towards favoring mixed-gender interactions.

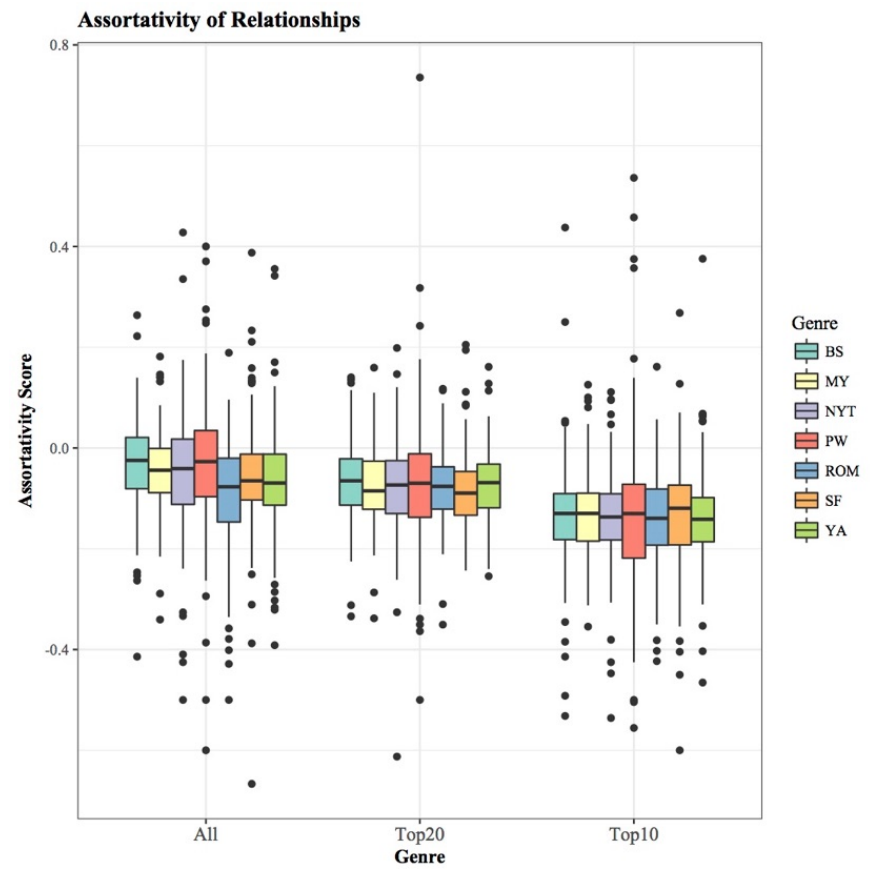

Figure 5. Assortativity scores by genre for character relationships; we see homophily (like with like) growing as we move into the higher ranks of character importance as well as very strong outliers in both directions. 


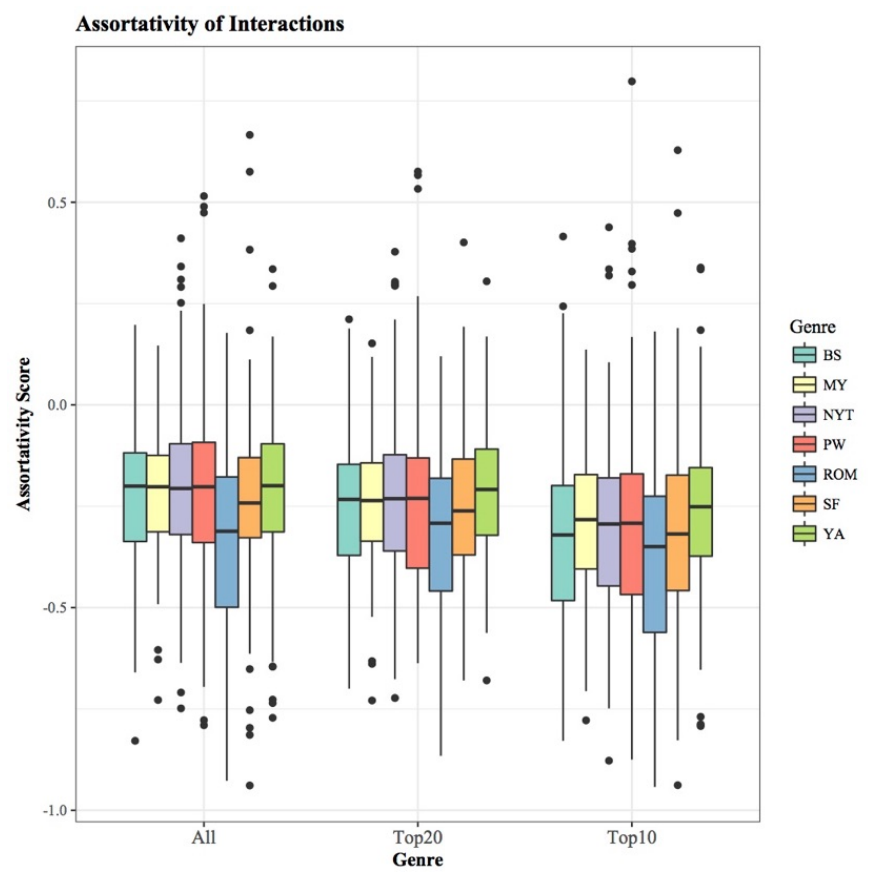

Figure 6. Assortativity scores by genre for character interactions-similarly, we see homophily growing as we move into the higher ranks of character importance and very wide distributions.

To measure the relationship between genre and assortativity, we use an analysis of variance test and find that there is no significant difference between the degree of relationship assortativity by genre for either the top 10 or top 20 characters $(\mathrm{F}(6,1078)=0.668, \mathrm{p}=0.67)$ and $(\mathrm{F}(6,1078)=1.32, \mathrm{p}=0.244)$, though there was for all characters $(\mathrm{F}(6,1078)=2.812, \mathrm{p}=0.01)$. For interactions, Romance scored significantly lower than all other genres across all subsets (indicating more mixed-gender pairs), while YA indicated a significantly higher level of same-gender pairs for the top twenty and ten characters. With Romance removed, there was a weakly significant difference in gender assortativity across all interactions $(\mathrm{F}(5,959=2.09, \mathrm{p}=0.06)$ and once YA was removed, the genres were not significantly different at the top-ten $(\mathrm{F}(4,840)=1.36, \mathrm{p}=0.247)$ or the top-twenty $(\mathrm{F}(4,840)=1.13, \mathrm{p}=0.34)$.

Finally, when we examine author gender we see that for all categories of both relationships and interactions, women authors use considerably more mixed-gender connections (once again, even with Romance removed). There is a distinct bias 
in the work of women writers towards depicting a more dissassortative social world, with a significantly greater amount of interactions between genders in novels written by women across all categories, except for those amongst the top 10 characters (Table 5). While women authors increase the number of women in the novel, they also increase the number of mixed-gender interactions, especially among the less prominent characters.

\begin{tabular}{llllll}
\hline Category & Set & Women & Men & $\mathrm{t}$ & $\mathrm{p}$ \\
\hline Relationships & All & $\mathrm{M}=-0.05, \mathrm{SD}=0.07$ & $\mathrm{M}=-0.03, \mathrm{SD}=0.17$ & -3.8 & 0.00016 \\
Relationships & Top 20 & $\mathrm{M}=-0.08, \mathrm{SD}=0.08$ & $\mathrm{M}=0.06, \mathrm{SD}=0.09$ & 3.34 & $0.0006^{*}$ \\
Relationships & Top 10 & $\mathrm{M}=-0.14, \mathrm{SD}=0.09$ & $\mathrm{M}=-0.13, \mathrm{SD}=0.11$ & -1.21 & 0.23 \\
Interactions & All & $\mathrm{M}=-0.23, \mathrm{SD}=0.16$ & $\mathrm{M}=-0.19, \mathrm{SD}-0.17$ & -3.8 & 0.00015 \\
Interactions & Top 20 & $\mathrm{M}=-0.26, \mathrm{SD}=0.17$ & $\mathrm{M}=-0.22, \mathrm{SD}=0.18$ & -3.07 & 0.002 \\
Interactions & Top 10 & $\mathrm{M}=-0.31, \mathrm{SD}=0.19$ & $\mathrm{M}=-0.13, \mathrm{SD}=0.13$ & -1.63 & $0.104^{\star}$ \\
\hline
\end{tabular}

Table 5. Comparison of assortativity scores between men and women authors.

\section{Antagonism}

Having established the relationship between gender and the larger network of interactions in our contemporary novels, we now want to move towards an understanding of the quality of those interactions. Here we explore a single variable, that of antagonism between two characters, which we operationalize as the average ratio of all positive to negative words associated with a given pair type per novel. Using the top twenty characters for each novel, we compare this ratio for men-only and women-only pairs. In essence, we want to know if the gender dynamic in novels impacts the likelihood of seeing more or less negativity between characters of the same gender.

Overall we found that women-pairs used significantly higher rates of positive vocabulary than pairs of men $(\mathrm{F}(1,2507)=24.14, \mathrm{p}=2.04 \mathrm{e}-07)$, though this difference from a reader's perspective is relatively weak. We see a five to seven percent increase in the ratio of positive words, which only amounts to less than a single word for a 10-11 word sentence (the broad average for a novel). Over the course of an entire novel this will indeed add-up (with thousands of character co-occurrences), but at the individual sentence or page level it only marks a slight shift. Genres exhibit these sentiment ranges in significantly different ways $(\mathrm{F}(6,2507)=2.85, \mathrm{p}=0.0058))$ with Bestsellers having a difference of 0.33 between men and women, and NYT novels having only a mean difference of 0.07 across gender pairs. Interestingly, author gender does not significantly impact 
the scores for men and women $(\mathrm{F}(1,2496)=1.427, \mathrm{p}=0.23)$, suggesting that these gendered differences in antagonism could be more closely tied to either narrative structures or the vocabulary tested than extradiegetic factors.

\section{Balance}

Studying pairs in novels gives us insights into an elementary character structure that falls along the binary lines of protagonism and antagonism, but also a sense of companionship, that is, when strong pairings in novels are not driven by conflict but by a sense of mutual support (the archetypal example for the novel might be Don Quixote and Sancho Pansa but one could think of many more). What it cannot capture are more complex relational structures that extend beyond the dyadic model of the pair. Here we want to introduce the idea of social balance, which examines the stability of triangular relationships within more complex network structures.

Social balance theory is a well-studied area in the social and cognitive sciences and dates back to the post-war research of Fritz Heider. ${ }^{24}$ It is premised on the notion that individuals seek out cognitive equilibrium in their social relationships such that they will attempt to balance out or disengage from unbalanced social configurations. As we can see in Fig. 8, balanced and unbalanced conditions are defined as the following four states:
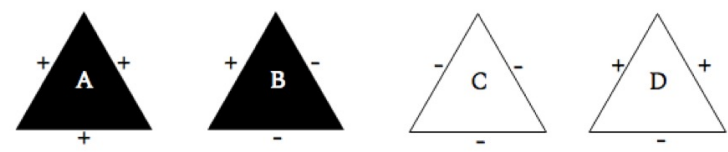

Figure 7. Models of social balance in social triangles.

\section{Balanced}

(a) when three characters inhabit mutually positive relationships with one another

\footnotetext{
${ }^{24}$ Fritz Heider, "Attitudes and Cognitive Organization,” The Journal of Psychology21 (1946): 107112 and Dorwin Cartwright and Frank Harary, "Structural Balance: A Generalization of Heider's Theory," Psychological Review 63 (1956): 277-293.
} 
(b) when two characters inhabit a positive relationship with each other and a mutually negative relationship with a third party ("mutual enemy")

\section{Unbalanced}

(c) when three characters all inhabit negative relationships with one another

(d) when a single character is in a positive relationship with two people who are in conflict with one another

Given these two possible states, our question is whether gender is a factor that is related to the overall prevalence of balanced states within novels. Do women participate significantly more often in balanced or unbalanced social cliques?

In order to test this, we look at the four possible gender configurations shown in Fig. 9 (MMM, WWW, MWW, MMW). To determine whether a relationship is positive or negative, we compare the average sentiment score of a given pair to the average sentiment score in the novel overall. We choose this to control for variations in word use across books and genres. If a given pair in a triangle scores a higher than average sentiment value for that book, then we label it as positive (and vice versa for lower than average). Conditioning on the triangles formed using the top ten characters per novel, we analyze 58,8831 triangles from our data set. The most common gender structure are triangles with one woman, while the least common are triangles with three women, which holds true for every genre. It is telling that the number of triangles with just two women, i.e. when there is a dominance of women in a transitive relationship, approximate the number of triangles containing three men. In other words, novels appear to compensate for partial social dominance by women with complete dominance by men. This corroborates both of our primary findings in section one, which suggest a tendency towards masculine dominance in novels that works in tandem with a heteronormative bias. In terms of balance, the majority (31,092 triangles) are balanced, and the remaining 27,739 triangles are imbalanced.

To begin to understand the ratio between these triangles, we calculate the overall ratio of balanced to imbalanced states within novels and find that this ratio is not consistent across all genres $(\mathrm{X} 2(6, \mathrm{~N}=58,831)=33.166, \mathrm{p}=9.743 \mathrm{e}-06)$. NYT reviewed novels represented one end of the spectrum, with a significantly higher ratio of balanced to imbalanced $(1.2 \mathrm{x})$ and Bestsellers on the other end, with a ratio of $1.07 \mathrm{x}$, exhibiting a greater investment in imbalanced social states (though still an overall preference for balanced states).

From this, we then looked at whether gender has an effect on the ratio of balanced 
to imbalanced triangles. Is it the case that certain gender configurations are associated with more or less social balance? We are asking whether the presence of a single woman produces more imbalance (mimicking some kind of evolutionary model of reproductive selection) or whether more women produce more balance, mirroring our findings above that women pairs are somewhat more conflict averse overall. When combining all genres, however, we do not see a significant difference in the ratio of balanced to imbalanced triangles when conditioning on the gender of the characters $\left.\left(\mathrm{X}^{2}(3, \mathrm{~N}=58,831)=1.41, \mathrm{p}=0.7024\right)\right)$. We also find that that author-gender does not influence the likelihood of the appearance of a balanced or imbalanced triangle $(\mathrm{X} 2(1, \mathrm{~N}=51,146)=0.09, \mathrm{p}=0.7613)$.

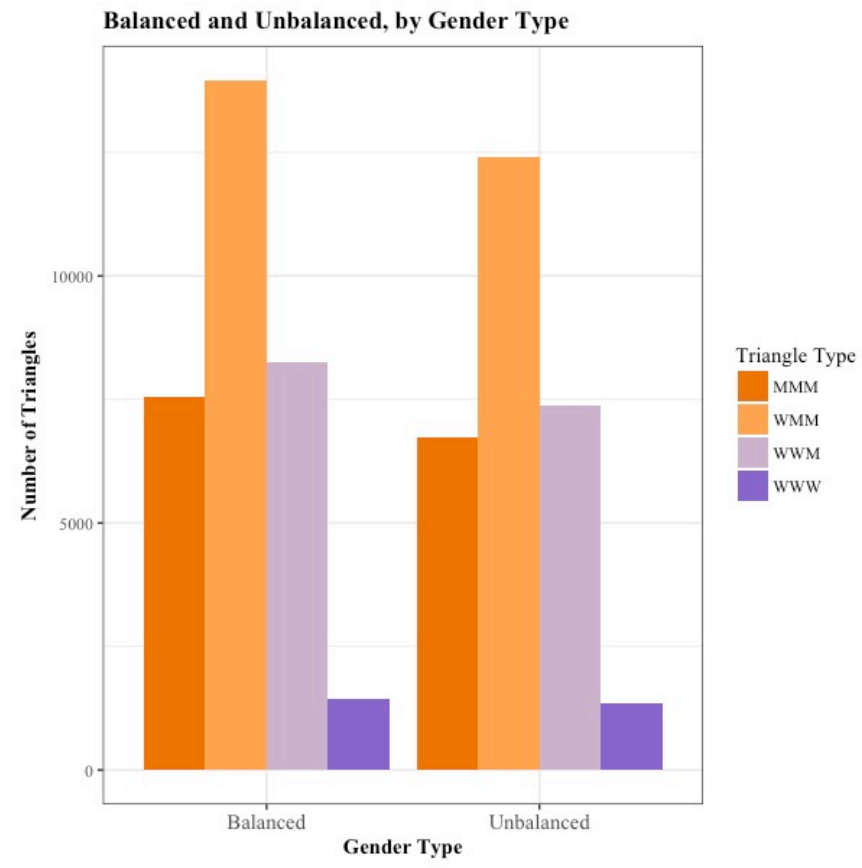

Figure 8. Balance and imbalanced social triangles by gender configuration.

In short, when it comes to social balance as indicated by pairwise sentiment scores, we see little indication that the character's gender has an effect on the state of social balance. Possible areas for further investigation would be testing the efficacy of the sentiment measures as well as whether taking a global measure of pairwise sentiment is the best approach. One could imagine that relationships evolve over the course of a novel and so an average of all interactions might miss important trends within the narrative. But for now, we see little evidence 
to suggest that contemporary novels generate social triangular conflict through character gender.

\section{Discussion}

Our research provides a preliminary investigation into the presence of gender bias in the social construction of character in contemporary novels. In terms of visibility, our measures suggest that (1) women are less visible than men at the level of individual entities; (2) that these ratios operate in highly predictable ways across different genres and books targeting different audiences; and (3) that author gender can reduce, but not erase, these inequalities.

As we discuss in our introduction, these effects have been found in a variety of media and cultural spheres, so it should come as little surprise that we have found similar effects within books. Nevertheless, we began with the implicit assumption that literature might be different. Book publication is cheaper than creating a Hollywood movie. The audiences for prizewinning fiction can be assumed to be quite different from the Hollywood blockbuster demographic. At the same time, fictional women are not subject to the real-world constraints that impact women's representation in the news, for example. But our measures show that fictional texts are subject to the same gestures and ratios of gender bias as other media with one notable exception: novel protagonist. In this case, we see how the contemporary novels in our data set promote gender equality at the level of the main character but then undo this in the rest of the novel's social universe. The consistency of this gender bias below the surface of the novel's protagonist suggests to us the way gender hierarchies are written into the latent codes of contemporary narrative structures. Women are not only less present in terms of their overall quantity within novels, but regardless of genre, cultural capital, or even readership age, the visible spaces they inhabit are shaped and sized in similar ways.

Given that these measures point to an overall decentralization of women, it is interesting to note that women authors actually account for the majority of our texts. In other words, with a more balanced sample in terms of author gender our findings would look even worse. The presence of women authors then does make a substantial impact on the presence of fictionalized women characters. Nevertheless, this rise is relative. Both genders still skew towards the overall visibility of fictional men. Author gender can control only the strength of the gender skew, 
not its existence in fiction. Increasing representation at the level of authorship will not completely solve the problem.

What are we to make of this? While it might be most straightforward to say that less visibility for women in novels is inherently negative, our measures on social connectivity complicate this notion. As assortativity measures indicate, women are highly unlikely to be paired with other women, but also seem to have a tendency towards less conflict when they do interact. On the one hand, it offers a positive portrait of women's relationships that instantiate a distinctively less antagonistic model of social interaction. On the other hand, not only does this conform to widespread stereotypes of women being more conflict averse, we also wonder whether it has an impact on notions of literary significance. If there is a latent belief that great novels are those where characters overcome some kind of social conflict, then relationships that are noticeably less conflict ridden-and also considerably rarer in general-will appear less “important." As Arthur Krystal writes in a recent piece that has since been adapted into a book-length reflection on the profession of literary criticism:

Fiction, speaking very generally, is about the individual in society, about the expectations and conflicts that color a life when an obdurate reality stands in the way of one's self-image or desires...The invisible centerpiece of every great novel is the protagonist's rebellion or coming to terms with his or her place in the scheme of things. ${ }^{25}$

While we would not want to take Krystal's ideas as representative of public or critical opinion on the novel, it does give us pause to wonder whether these kinds of definitions of literary greatness privilege a certain type of social gender dynamic that works against the interests of women, as both writers and fictional characters.

What is most prominent from these findings, and indeed across our connectivity measures, is that women are far less likely to be put in contact with other women, regardless of the content or meaning of the conflict between them. In particular, we see that in our measure of gender assortativity, mixed-pairings are not only more common, but are so in highly standardized and deliberate rates across genres. While research suggests that real-world networks become more disassortative as people age (starting highly gendered in childhood and adolescence), they tend to remain more same-gendered, especially for women, indicating the extent to which novelistic social networks depart from real-world ones. ${ }^{26}$

\footnotetext{
${ }^{25}$ Arthur Krystal, "The Novel as a Tool for Survival," The Chronicle of Higher Education (2016).

${ }^{26}$ For an overview of social network effects, see David Laniado, Yana Volkovich, Karolin Kappler and Andreas Kaltenbrunner, "Gender homophily in online dyadic and triadic relationships," EPJ Data Science (2016) 5:19 DOI 10.1140/epjds/s13688-016-0080-6; Kristine J. Ajrouch, Alysia Y. Blan-
} 
Taken together, this suggests that social interactivity in contemporary novels is conditioned by a concerted effort to signal and foreground heteronormative social relations. Here, women authors tend to exaggerate the pattern, relying more heavily than authors that are men on mixed-gender connections, even when we exclude Romance. The bias against women characters in novels is complemented by a bias towards heteronormative social structures.

\section{Limitations}

Before we conclude, it is important to note several limitations to our research. In addition to the limitations with respect to our dataset discussed at the opening of our essay, we also make three conceptual simplifications that give us pause when it comes to understanding our findings. First, in our assessment of social connections in the novel we rely on a grammatical model of sentence co-occurrence, which is not the same as capturing actual social interactions in the diegetic universe of the novel. Agarwal et al. have proposed a productive framework for thinking about automatically detecting and classifying social interactions, but the reliability of detection continues to be quite low. ${ }^{27}$ While studying co-occurrence involves a simplification of novelistic narrative, it has the advantage of providing a coherent and consistent unit of analysis, similar to the use of "scenes" as

don, and Toni C. Antonucci, "Social networks among men and women: The effects of age and socioeconomic status," The Journals of Gerontology Series B: Psychological Sciences and Social Sciences 60. 6 (2005): 311-317. For research on social networks and cellphones, see Tasuku Igarashi, Jiro Takai, and Toshikazu Yoshida, "Gender differences in social network development via mobile phone text messages: A longitudinal study," Journal of Social and Personal Relationships22.5 (2005): 691-713. For social network effects and aging, see Diana Shye, John P. Mullooly, Donald K. Freeborn, and Clyde R. Pope, "Gender differences in the relationship between social network support and mortality: a longitudinal study of an elderly cohort," Social Science \& Medicine41. 7 (1995): 935-947. For a study of online gaming communities, see Michael Szell and Stefan Thurner, "Measuring social dynamics in a massive multiplayer online game," Social Networks32.4 (2010): 313-329.

${ }^{27}$ See Apoorv Agarwal, Sriramkumar Balasubramanian, Anup Kotalwar, Jiehan Zheng and Owen Rambow, "Frame Semantic Tree Kernels for Social Network Extraction from Text," Proceedings of the 14th Conference of the European Chapter of the Association for Computational Linguistics (2014): 211219. They report a best F1 score of 58.5. Other proposed models use dialogue as a form of interaction. See David K. Elson, Nicholas Dames, and Kathleen R. McKeown, "Extracting social networks from literary fiction," in Proceedings of the 48th annual meeting of the association for computational linguistics, Association for Computational Linguistics, (2010), 138-147; and Michael C. Waumans, Thibaut Nicodème, and Hugues Bersini, “Topology analysis of social networks extracted from literature," PloS one 10.6 (2015): e0126470 
boundaries in the study of social networks in drama. ${ }^{28}$ At the same time, while our models take into account different unit-levels (singles, pairs, triads), we do not address time-based features of network development. We surmise that these are important to add nuance to the question of narrative social networks though we are less certain how temporality and gender might intersect.

Second, we operationalize gender through the use of a binary structure. We understand that this does not reflect the reality and diversity of gender identity, neither of people nor characters in fiction. Nevertheless, our aim here is the study of linguistic, not real entities, and these linguistic entities are overwhelmingly signaled to readers through binary terms in the form of pronouns, which comprise roughly $75 \%$ of all character mentions. There can be many types of masculinity or femininity communicated through nuanced semantic profiles of characters. However, given that three-quarters of the references to characters in books are through pronouns like "he" and "she" that necessarily signal a binary, characters are heavily associated with notions of either manhood or womanhood. Bringing attention to this practice through characters allows us to make explicit the otherwise latent ways in which gender is being mobilized and hierarchized within novels. At the same time, we see future work engaging with questions of multiple gender identities surrounding the practice of characterization. ${ }^{29}$

Third, in only conditioning on character gender, we isolate gender bias from other forms of oppression and privilege. In her "Demarginalizing the Intersection of Race and Sex: A Black Feminist Critique of Antidiscrimination Doctrine, Feminist Theory and Antiracist Politics," Kimberle Crenshaw coins the phrase "intersectionality" to describe the position of women of colour, who face systemic oppression from both their gender and racial identity, at once. Turning all women's experience into a single form of gendered oppression often further "marginalizes those [women] who are multiply-burdened." ${ }^{30}$ Scholars and activists have since expanded Crenshaw's concept to investigate how race and gender can further intersect with factors such as class, sexuality, ability, and ethnicity. Together, this work rightfully challenges the possibility of a singular, universal feminism or womanhood, and instead elevates the voices and concerns of intersectional women. ${ }^{31}$ In our research, we are not yet able to control for these

\footnotetext{
${ }^{28}$ See Mark Algee-Hewitt, "Distributed Character: Quantitative Models of the English Stage, 15001900," New Literary History (2017).

${ }^{29}$ See Mandell, Debates in Digital Humanities, forthcoming. Kiriloff et al. on studying outliers within gendered norms.

${ }^{30}$ Kimberle Crenshaw, "Mapping the margins: Intersectionality, identity politics, and violence against women of color," Stanford law review(1991): 1241-1299.

${ }^{31}$ For an exploration of the limits and applications of intersectional feminism, see Nira Yuval-Davis, "Intersectionality and feminist politics," European Journal of Women's Studies13, no. 3 (2006): 193209, Carole Boyce Davies, "Feminist consciousness and African literary criticism," Women in Culture:
} 
other intersecting factors that might differentiate bodies, and thus our model necessarily flattens the category of "women" to encompass the intersecting identities and privileges that that label can contain. Future research is needed to parse out and better conceptualize what "womanhood" is in each of our aggregated cases, and how its oppression functions unevenly across different categories.

\section{Conclusion}

As a way of moving forward, we see two ways of thinking about these gendered patterns in the novel. The first is diagnostic, the second what we call advocational. ${ }^{32}$ The relative independence of gender norms from questions of genre or audience suggest that there is indeed something "systemic" about this problem. Indeed, even the effect of author gender that we are seeing does not entirely mitigate the problem. It suggests that there are unstated norms and expectations across the publishing industry that generate surprisingly consistent outcomes, ones that mirror larger social inequalities. It forces us to question what we might call the escapist theory of reading, where a narrative network is a space where readers can elude the demands and constrictions of real life. In her article "Reading is Not Eating," Janice Radway discusses how early women's fiction provided a respite to its readers, because women could extricate themselves from the demands of their circumstances, the demands of being a mother or daughter or wife or sister, while they were reading. ${ }^{33}$ As Mrs. Ramsay, one of the prototypical fictional reading women, asks herself while reading a book in To the Lighthouse, "If he wanted her to wake, she really would, but otherwise, might she go on sleeping, just a little longer, just a little longer? She was climbing up those branches, this way and that, laying hands on one flower and then another." ${ }^{34}$ The isolated reader is imagined to find respite in a book. But if the worlds that women readers continue to enter replicate the very networks one is trying to escape, how far can they really go?

\footnotetext{
An Intersectional Anthology for Gender and Women's Studies 1.2 (2016): 120; and Leila Ahmed, Women and Gender in Islam: Historical Roots of a Modern Debate (Yale University Press, 1992).

${ }^{32}$ Here we take up the suggestion of Alexis Lothian and Amanda Phillips to use digital methods to engage with the "processes and possibilities of social and cultural transformation." See Alexis Lothian and Amanda Phillips, "Can Digital Humanities Mean Transformative Critique?" E-Media Studies 3.1 (2013): DOI:10.1349/PS1.1938-6060.A.425.

${ }^{33}$ Janice Radway, "Reading is not eating: Mass-produced literature and the theoretical, methodological, and political consequences of a metaphor," Book Research Quarterly 2, no. 3, (September 1989): 7-29.

${ }^{34}$ Virginia Woolf, To the Lighthouse (Orlando: Harcourt, 2005).
} 
This latter realization is a sobering one, yet it is also an opportunity. Quantifying these norms allows us to better understand what from a stylistic point of view would need to change in order to make fiction something that rewrites rather than replicates reality. In addition to advocating for better "representation" in terms of authorial identity-more real women assuming a more prominent place within publishing - we can also advocate for better representation in terms of the fictional identities that populate the novels readers consume and potentially escape into. The research used here allows us to see what the specific conditions within novels are that need to change in order to undo these norms. It can provide a concrete template for advocacy. Making fiction less "real" may not necessarily change reality. But it at least gives us an alternative, an imaginative landscape where things might be otherwise.

This research was supported by a grant from the Social Sciences and Humanities Research Council of Canada.

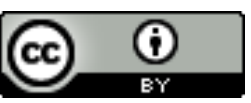

Unless otherwise specified, all work in this journal is licensed under a Creative Commons Attribution 4.0 International License. 